\title{
Implications of Direct Healthcare Professional Communication in Egypt: barriers and preferences of Health Care Professionals
}

\author{
Mai A. Faied ${ }^{a}$, Lamia M. El Wakeel ${ }^{* b}$, Amr A. Saad ${ }^{c}$ Nagwa A. Sabri $^{\text {b }}$ \\ ${ }^{a}$ The Egyptian Pharmaceutical Vigilance Center, Central Administration for Pharmaceutical Affairs, Ministry of \\ Health, Cairo, Egypt \\ ${ }^{b}$ Clinical Pharmacy Department, Faculty of Pharmacy, Ain Shams University, Cairo, Egypt \\ ${ }^{c}$ The National Organization for Drug Control And Research (NODCAR), Cairo, Egypt
}

\begin{abstract}
Direct Healthcare Professional Communication (DHPC) is essentially distributed for fast communication of new serious drug safety information to healthcare professionals (HCPs). However, the use of this tool concerning the knowledge and preferences of HCPs has never been evaluated in Egypt. This study aimed to evaluate the HCPs' knowledge, preferences, and barriers to the use of DHPC in Egypt. A crosssectional study of a random sample of 254 HCPs surveyed via face-to-face interviews to assess the Egyptian HCPs' awareness of DHPC, the preferences, and barriers that affect its use. Among the 297 approached HCPs, only 254 accepted to participate with a response rate of $85.5 \%$, including $(50 \%$ internists, $22.83 \%$ cardiologists, $11.02 \%$ neurologists, $11.02 \%$ pediatricians, and $5.12 \%$ from other specialties). Most HCPs were not familiar with DHPC $(\mathrm{N}=254,61.8 \%)$. One-third of the visited HCPs who were aware of concerned drugs' risk(s) got their information from DHPC (N=149, 36.9\%). HCPs preference for communication channel was highest for meetings $(\mathrm{N}=254,65.7 \%)$ and least for newsletters $(\mathrm{N}=254,28 \%)$. HCPs reported barriers to reading DHPC included; busy schedule $(\mathrm{N}=254$, $47.6 \%)$, mistrusted source $(\mathrm{N}=254,24.4 \%)$, view as a marketing tool $(\mathrm{N}=254,21.7 \%)$, invaluable information ( $\mathrm{N}=254,9.8 \%)$ and disbelief $(\mathrm{N}=254,7.5 \%)$. The DHPC did not reach the target HCPs most of the time, but when received, it was successful in conveying the required message to the target HCPs. Multiple barriers were identified that negatively impacted the success of DHPC. It is recommended to use other electronic communication methods to enhance the reachability of the current method (DHPC).
\end{abstract}

Keywords: Healthcare Professionals; Direct Healthcare Professional Communication; Egyptian pharmaceutical Vigilance Center; Ministry of Health

\footnotetext{
*Correspondence | Lamia M. El Wakeel; Department of Clinical Pharmacy; Faculty of Pharmacy, Ain Shams University, Cairo, Egypt. Email: 1amywak@yahoo.com

Citation | Faied MA, El Wakeel LM, Saad AA, Sabri N A, 2019. Implications of Direct Healthcare Professional Communication in Egypt: barriers and preferences of Health Care Professionals. Arch Pharm Sci ASU 3(2): 268-276

DOI: $10.21608 /$ APS.2019.15297.1007

Print ISSN: 2356-8380. Online ISSN: 2356-8399.

Received 18 August 2019. Accepted 10 October 2019.

Copyright: ${ }^{\circledR} 2019$ Faied et al. This is an open-access article licensed under a Creative Commons Attribution 4.0 International License (CC BY 4.0), which permits unrestricted use, distribution, and reproduction in any medium, provided the original author(s) and source are credited. Published by: Ain Shams University, Faculty of Pharmacy
}

\section{INTRODUCTION}

For any medicinal product to be authorized, its benefits should outweigh its risks in the specified indication(s) [1]. Pharmacovigilance starts during clinical trials and continues after the drug is released into the market. However, due to 
the various limitations, the complete benefit-risk profile of new drugs can never be achieved through the pre-approval phase of clinical trials, so, with the large scale use of a drug in real life, new serious safety issues may be raised [2].

Thus pharmaceutical companies should continuously monitor their medicinal product's safety and inform the health authority of any changes in the known drug safety profile that might affect the risk-benefit balance of the product, and affirm that the product information always remains updated [3]. In certain cases when a serious safety issue is raised necessitating immediate dissemination of information, Direct Health Care Professional Communication (DHPC) distribution can be requested from the related pharmaceutical company [1]. DHPCs are mainly used to communicate urgent new serious safety information to the target Health Care Professionals (HCPs) on time [4].

There is unclear data about HCPs awareness of DHPC [5, 6]. Besides, the successful delivery of DHPC to HCP, its clarity, and its effectiveness in changing the prescribing practice of targeted HCPs is still questionable [7]; few studies revealed that DHPCs were suitable in conveying the required message and affecting the HCP's behavior [8-10], however, the majority of conducted studies showed several defects in such risk communication tool, ranging from the fact that it did not actually reach the target HCPs [4, 11], to its poor ability in communicating the required data [12, 13]. Hence, some studies recommended the use of an additional method with DHPC for better risk communication. $[4,11$, $14,15]$. To date, the use of DHPC has never been evaluated in Egypt. This study was designed to assess the HCPs' knowledge, preferences, and barriers to the use of DHPC in Egypt.

\section{MATERIAL AND METHODS}

This is a cross-sectional study evaluating the use of DHPC by HCPs who should have already received the tested DHPC(s).

\subsection{Ten different DHPCs of those distributed before the study time were selected (Table 1 ).}

\subsection{A general survey form was used}

It was adapted from a survey used in an earlier study [11]. It included open-ended and closed-ended questions that assessed knowledge, preferences of HCPs regarding DHPC and barriers hindering its success, where:

$>\quad$ The knowledge of HCPs was assessed through several questions including:

○ Have you ever seen a DHPC?

○ Do you read the DHPCs you receive?

- Do you visit the Egyptian Pharmaceutical Vigilance Center (EPVC) website for specific information on drug safety issues?

- Are you aware of the "safety issue" which was sent on the date of DHPC distribution?

○ If yes; How did you receive this information (DHPC; EPVC's website; Media; Specialist journal; electronic mailing/internet; other, namely)?

For improving information delivery, the HCPs' preferences were assessed using a 10 point Likert scale, ranging from (1) not useful at all to (10) very useful, (evaluated as follows: $<5$ is considered not useful, 5-7 is considered moderately useful, $>7$ is considered highly useful), where the following was investigated:

W Which of the following information channels (by hand, e-mail, post, newsletter, social media, and meetings/events) do you think are suitable for the fast delivery of drug safety information?

Which of the following senders (EPVC, Marketing Authorization Holder (MAH), or both) 
do you think are suitable for fast information about new drug safety issues?

$\circ \quad$ Which of the following factors (source the novelty of drug - the simplicity of information -interest in the topic - length of DHPC - the importance of information - all) affect your interest in reading the DHPC?

Barriers attributed to decreasing the DHPC effectiveness included: (busy schedule-do not believe in it-do not believe it contains valuable information - do not trust its sourcethink it is a marketing tool)

\subsection{Population sampling}

A sample of 254 HCPs - working in Cairowere randomly selected from the whole group of HCPs who should have received the test DHPCs $(n=3979)$ using Raosoft sample size calculator [16], with $90 \%$ confidence interval. Such sample was surveyed through a face-to-face meeting during the period (from June 2016 to January 2017).

The study protocol was revised and approved by the Committee of Ethics, Faculty of Pharmacy, Ain Shams University (serial number: 83).

Table 1: Listing of the selected DHPC evaluated in the study

Title of DHPC

CellCept $^{\circledR}$ (mycophenolatemofetil): teratogenic risk-important new pregnancy prevention advice for women and men

Valproate risk of abnormal pregnancy outcome

New contraindications and warnings for the use of Angiotensin II receptor blockers (ARBs) or Angiotenisn Converting Enzyme Inhibitors (ACEIs) in combination with Aliskerin containing medicines

Combined hormonal contraceptives: be aware of the difference in risk of thromboembolism between products, the importance of individual risk factors and remain vigilant for signs and symptoms

Special warnings and precautions for use of products containing Tenofovir DF, namely Viread or Truvada

Intrauterine contraceptives: Update on risk of uterine perforation

Domperidone: new recommendations to minimize the cardiac risks

Association of Clopidogrel $\left(\right.$ Plavix $^{\circledR}$ ) with acquired hemophilia

Serious and life-threatening cases of symptomatic bradycardia as well as one case of fatal cardiac arrest reported with coadministration of Amiodarone with either Sofosbuvir in fixed dose combinations/ combinations with another Direct Acting Antiviral drug for example (but not restricted to) Ledipasvir, Daclatasvir or Simeprevir respectively.

Denosumab 60mg (Prolia ${ }^{\circledR}$ ): Updated information to minimize the risk of osteonecrosis of the jaw and hypocalcaemia

\section{Target specialty}

Internists (Nephrologists)

Neurologists

Cardiologists and Internists

Gynecologists

Internists (Hepatologists)

Gynecologists

Cardiologists, Internists, Pediatricians

and General Practitioners (GPs)

Cardiologists and Internists

Internists (Hepatologists)

Internists 


\subsection{Statistical analysis}

Data were presented in structured data entry forms. Statistical analysis was done using IBM(C) SPSS $\odot$ Statistics version 22 (IBM $\odot$ Corp., Armonk, NY, USA). Categorical data were expressed as frequency and percentage. Pearson's Chi-square test or Fisher's exact test was used to examine the relationship between qualitative variables. All tests were two-tailed. A p-value < 0.05 was considered statistically significant.

\section{RESULTS}

Around 297 Egyptian HCPs were visited for the survey conduction. The overall response rate was $85.5 \%(\mathrm{~N}=254$, including $50 \%$ internists, $22.8 \%$ cardiologists, $11 \%$ neurologists, $11 \%$ pediatricians, and $5.1 \%$ from other specialties). The survey flow chart is represented in (Fig. 1). The demographic data of participating HCPs is represented in Table 2.

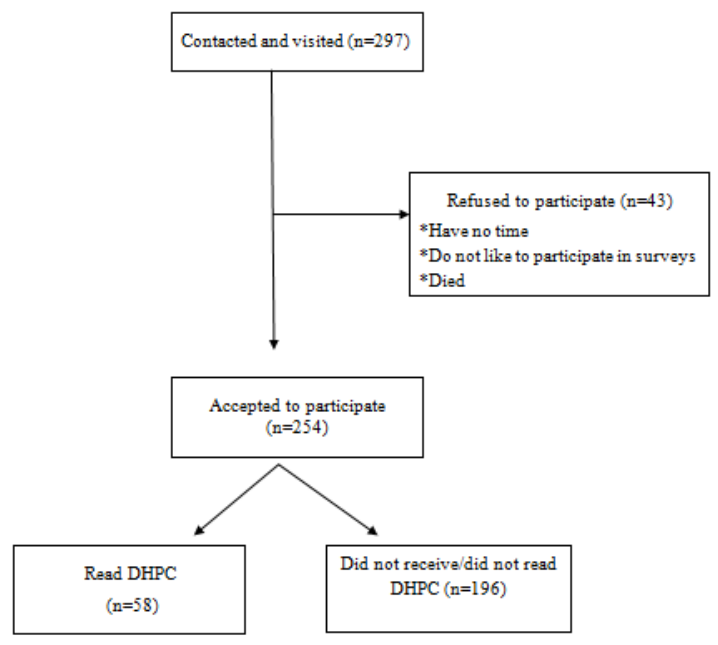

Fig. 1. Survey flow chart (Note: DHPC: Direct Health Care Professional Communication).

Table 2. Demographic data of the participants

\begin{tabular}{cccccccc}
\hline & & $\begin{array}{c}\text { Internists } \\
(\mathrm{N}=127)\end{array}$ & $\begin{array}{c}\text { Cardiologists } \\
(\mathrm{N}=58)\end{array}$ & $\begin{array}{c}\text { Neurologists } \\
(\mathrm{N}=28)\end{array}$ & $\begin{array}{c}\text { Pediatricians } \\
(\mathrm{N}=28)\end{array}$ & $\begin{array}{c}\text { Others \# } \\
(\mathrm{N}=13)\end{array}$ & $\begin{array}{c}\text { Total } \\
(\mathrm{N}=254)\end{array}$ \\
\hline & Male & $104(81.9 \%)$ & $54(93.1 \%)$ & $21(75 \%)$ & $20(71.4 \%)$ & $9(69.2 \%)$ & 208 \\
Gender; n (\%) & Female & $23(18.1 \%)$ & $4(6.9 \%)$ & $7(25 \%)$ & $8(28.6 \%)$ & $4(30.8 \%)$ & $46(18.1 \%)$ \\
& $<10$ & $5(3.9 \%)$ & $7(12.1 \%)$ & zero & zero & $1(7.7 \%)$ & $13(5.1 \%)$ \\
Experience & $10-20$ & $23(18.1 \%)$ & $14(24.1 \%)$ & $4(14.3 \%)$ & $8(28.6 \%)$ & $4(30.8 \%)$ & $53(20.9 \%)$ \\
years; n (\%) & $21-30$ & $47(37 \%)$ & $19(32.8 \%)$ & $14(50 \%)$ & $11(39.3 \%)$ & $2(15.4 \%)$ & $93(36.6 \%)$ \\
& $>30$ & $52(41 \%)$ & $18(31 \%)$ & $10(35.7 \%)$ & $9(32.1 \%)$ & $6(46.1 \%)$ & $95(37.4 \%)$ \\
Scientific degree; & Bachelor & $2(1.6 \%)$ & zero & zero & zero & $5(38.5 \%)$ & $7(2.8 \%)$ \\
M (\%) & Master & $31(24.4 \%)$ & $18(31 \%)$ & $1(3.6 \%)$ & $11(39.3 \%)$ & $3(23 \%)$ & $64(25.2 \%)$ \\
& MD & $94(74 \%)$ & $40(69 \%)$ & $27(96.4 \%)$ & $17(60.7 \%)$ & $5(38.5 \%)$ & $183(72 \%)$ \\
\hline
\end{tabular}


The survey questions assessed the knowledge and preferences of HCPs toward DHPC and barriers hindering its use with consideration to the differences in; specialties, experience years, scientific degrees, and health institutions, as follows:

\subsection{Assessment of HCP's knowledge}

The higher percentage of HCPs were not familiar with DHPC $(\mathrm{N}=254,61.8 \%)$, except for the neurologists who showed a significantly high awareness of DHPC $(\mathrm{N}=28,64.3 \%, \mathrm{p}=0.030)$. There was a significant difference $(\mathrm{p}=0.018)$ in physician agreement to read the received DHPC, where HCPs having Medical doctorate (MD) degree showed the highest approval $(\mathrm{N}=183$, $90.7 \%$ ) versus those with a bachelor degree who showed the lowest approval $(\mathrm{N}=7,57.1 \%)$. Most of the HCPs had never heard about the Egyptian Pharmaceutical Vigilance Center (EPVC) $(\mathrm{N}=$ $254,89.8 \%$ ). Around half of the tested sample $(\mathrm{N}=254,58.7 \%)$ stated that they were aware of concerned safety issues. There was a significant difference $(p<0.001)$ between the various HCP specialties with regards to their awareness of safety information communicated through DHPC, where the neurologists showed the highest awareness $(\mathrm{N}=28,89.3 \%)$ versus cardiologists who showed the lowest awareness $(\mathrm{N}=58,44.8 \%)$. Around one-third of the visited HCPs who were aware of the concerned drug's $\operatorname{risk}(\mathrm{s})$ got such information from the DHPC $(\mathrm{N}=$ 149, 36.9\%) (Fig. 2.).

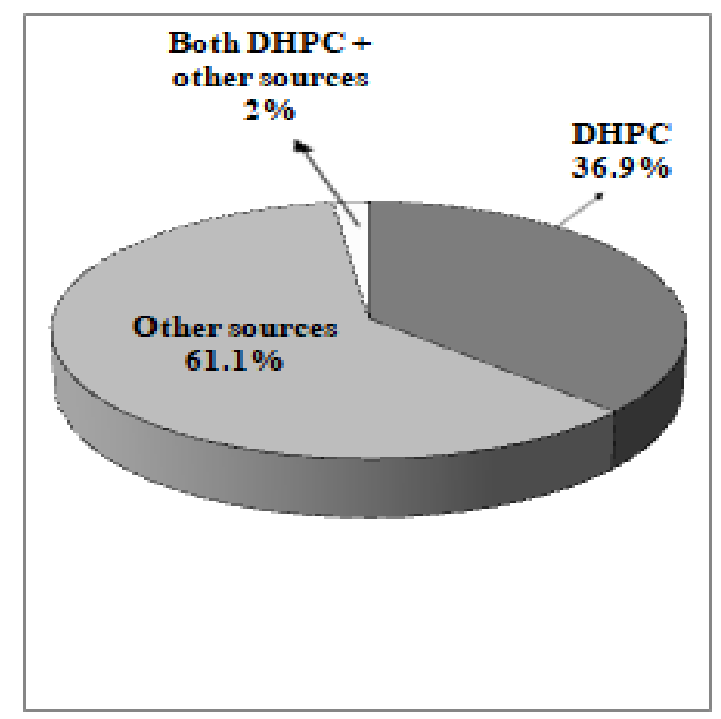

Fig. 2. Sources of Healthcare professionals' (HCP) awareness of concerned drug risks

The previous figure represents the participation of DHPC in spreading drug safety data to HCPs versus other sources of information like (scientific journals, media, colleagues, books, literature, and internet...), where it was found that HCPs got their information about drug safety from the other sources $(\mathrm{N}=149,61.1 \%)$ rather than from the Direct Health Care Professional Communication (DHPC) $(\mathrm{N}=149$, $36.9 \%$ ).

\subsection{Assessment of HCP's preferences for better risk communication and barriers hindering DHPC effectiveness}

Most HCPs selected multiple channels for fast delivery of drug information; the preferred channels were: meetings $(\mathrm{N}=254,65.7 \%)$, emails $(\mathrm{N}=254,61.8 \%)$, hand $(\mathrm{N}=254,61 \%)$, social media $(\mathrm{N}=254,53.1 \%)$, post $(\mathrm{N}=254$, $31.1 \%)$, and finally the least was newsletters $(\mathrm{N}=$ $254,28 \%)$. The majority of HCPs $(\mathrm{N}=254$, $72.4 \%$ ) preferred safety information to be issued by both EPVC and Pharmaceutical companies/MAHs (Fig. 3). 


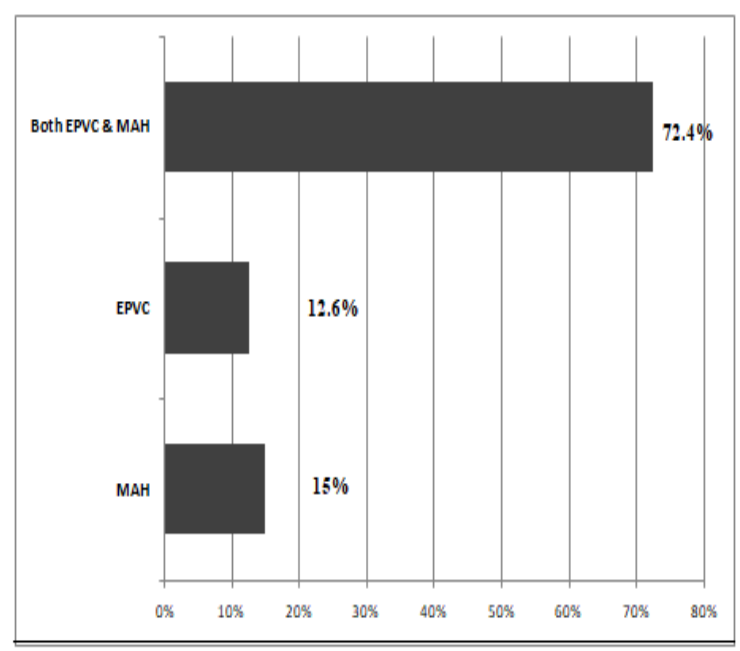

Fig. 3. Senders selected by HCPs for fast delivery of drug safety information

Fig. 3. represents the preference of HCPs with regard to the sender of information, where, receiving drug safety information from both EPVC and MAH is the most preferred by HCPs $(\mathrm{N}=254,72.4 \%)$ compared to MAH alone $(\mathrm{N}=$ $254,15 \%)$ or EPVC alone $(\mathrm{N}=254,12.6 \%)$.

Most of the HCPs clarified that multiple factors affected their interest in reading DHPC, as follows: importance of information $(\mathrm{N}=254$, $78 \%)$, interest in the topic $(\mathrm{N}=254,76 \%)$, source of information $(\mathrm{N}=254,72 \%)$, simplicity of information $(\mathrm{N}=254,68.9 \%)$, length of DHPC $(\mathrm{N}=254,60.2 \%)$, and finally, the least was novelty of the drug $(\mathrm{N}=254,59.8 \%)$. Multiple barriers were identified that hindered HCPs from reading a DHPC, the most prominent was "busy schedule" $(\mathrm{N}=254,47.6 \%)$ and the least was "disbelief in it" (N=254, 7.5\%) (Fig. 4).

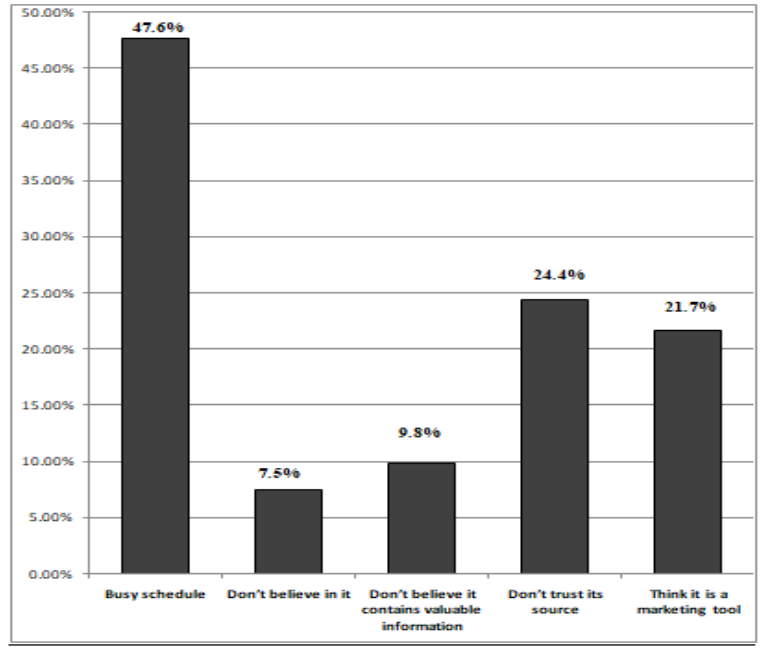

Fig. 4. Barriers that hinder Health Care Professionals (HCPs) from reading Direct Health Care Professional Communication (DHPC)

Fig. 4 represents the barriers that hinder HCPs from reading a DHPC, where the main barrier hindering effectiveness of DHPC was HCPs' busy schedule $(\mathrm{N}=254,47.6 \%)$ followed by their mistrust in DHPC source $(\mathrm{N}=254,24.4 \%)$, HCPs' thought that DHPC is a marketing tool $(\mathrm{N}=254,21.7 \%)$, think it does not contain valuable information $(\mathrm{N}=254,9.8 \%)$ and finally disbelief in it $(\mathrm{N}=254,7.5 \%)$.

\section{DISCUSSION}

In Egypt and Europe, DHPC can be distributed by $\mathrm{MAH}$ or medical authority (in special cases), to inform HCPs of the need to take certain actions or adapt their practices concerning a medicinal product $[\mathbf{1}, \mathbf{1 7}]$. DHPC is prepared by MAH in cooperation with the national medicines authority. Agreement between these two parties should be reached before the MAH starts the DHPC distribution. The agreement should include the DHPC content, distribution list specifying the target HCPs, the distribution mechanism, and the timeframe [1]. However, in Ghana, a Dear Healthcare Professional (DHP) letter is a correspondence usually in the form of a mass mailing from $\mathrm{MAH}$ of the medicinal 
product or a regulatory authority addressed to doctors, pharmacists, nurses and other health workers regarding important new safety information $[4,18]$. This study aimed to assess the HCPs' preferences and knowledge of DHPC and to detect barriers hindering the success of DHPC in Egypt. Three other studies were performed for a similar purpose, one in Netherland [11], another in Ghana [4] and a recent one conducted in nine European countries [14]. In the current study, HCPs of different specialties (internists, cardiologists, neurologists, pediatricians, and other specialties), with different experience years $(<20$ and $\geq 20$ years), several scientific degrees (Bachelor, Masters and Medical Doctorate (MD) degree), worked at different health institutions (university hospitals, educational institutes, and ministry of health) were surveyed through face-to-face interview. Around sixty-two percent $(\mathrm{N}=254,61.8 \%)$ of surveyed HCPs stated they have never seen a DHPC; this percentage is much greater than that reported in earlier studies; in the Netherland study [11], only $16 \%$ of respondents were not familiar with DHPC, while in the United States (US) [12] around 18\% of respondents reported never receiving a DHPC. The discrepancy in the awareness of HCPs with DHPC in Egypt versus the US and Europe could be attributed to the relative new initiation of pharmacovigilance services in Egypt compared to other countries and the necessity for more efforts to raise awareness of HCPs with DHPC.

Around half of the tested sample ( $\mathrm{N}=254$, $58.7 \%$ ) declared that they were aware of concerned safety issues, which was a comparable percentage to that found in Netherland study [11], where the awareness of most HCPs with all four safety issues ranged from (56\% to $88 \%$ ), while a higher percentage of $(92 \%)$ was detected in the European study [14]. Generally in the current study, for the ten tested DHPCs, the sources of such information varied between
HCPs where DHPC comprised ( $\mathrm{N}=149,36.9 \%)$ compared to other sources (conferences, media, journals, colleagues, books and internet) that comprised $(\mathrm{N}=149,61.1 \%$. $)$ This was in agreement with another study that revealed that HCPs use multiple sources other than DHPC to be updated with drug safety information [11]. On the other hand, in the Netherland study (2012) [11], professional journals were the major source of concerned information (59\%), followed by DHPC (49\%). Later, in 2018, the European study showed that the DHPC was the main source of information followed by messages on websites or in a newsletter or medical journal [14]. This highlights the urgent need for improving the current risk communication method by using additional/complementary sources of information together with DHPC in Egypt. Moreover, the increase in familiarity with HCPs with DHPC in Europe shows that intensifying efforts following the European path can help improve the risk communication method (DHPC).

The current study showed limited awareness of HCPs $(\mathrm{N}=254,99.2 \%)$ to EPVC and its website. Similarly, in the United Kingdom (UK), only around $20 \%$ of HCPs declared they were aware of Medicines and Healthcare products Regulatory Agency (MHRA), while in Canada, a higher percentage of $38 \%$ were aware of drug safety advisories published on the website of Health Canada [11]. Regarding the preferred information sender, in an earlier study [11], HCPs preferred to receive drug safety information either from Dutch Medicines Evaluation Board (MEB) or from their own professional associations, while in the current study, they preferred receiving such information from both Ministry of Health (MOH) and pharmaceutical companies simultaneously, as a kind of information reinforcement and bias exclusion. Regarding the preferred information channels, the majority of HCPs preferred to receive safety information through different 
channels; meetings, e-mails, hand, social media, post, and finally newsletters. Similarly, in Netherland [11], HCPs preferred to receive information mainly through e-mails. While in Ghana [4], and SMS to their mobile phones was the most preferred channel. In the current study, meetings were the most preferred channels, as they allowed face-to-face interaction and discussion, followed by electronic channels (email) which are a friendly, timesaving method for most HCPs. Moreover, some HCPs recommended that EPVC post any important drug safety data on the facebook pages or send message/e-mail to related HCP's categories. Other HCPs suggested that $\mathrm{MOH}$ distributes a brief letter reflecting the main drug safety issue to the clinical pharmacy department of each hospital, who will consequently pass such new information to related HCPs in their organization. This was previously recommended in the Netherland study [11], where physicians rated pharmacists as an alternative source of safety information, clarifying that the active involvement of these groups as intermediates in the risk communication process is an important step to support the process. Finally, regarding HCPs interest in reading a DHPC, the current study identified that the source, importance of information and interest in the topic were the main factors affecting HCPs interest in reading a DHPC. Besides, various barriers were identified from HCPs interviews that hinder the effectiveness of DHPC; the most prominent of which was "busy schedule" and the least was "disbelief in it".

\section{CONCLUSION}

Many times, the DHPC did not reach the target HCPs, but when received, it was clear and useful in conveying the required message to the target HCPs. The "Importance of information" and "interest in the topic" were the main triggers that affect HCP's interest in reading a DHPC, on the other hand, having a "Busy schedule" and "distrust in the source of DHPC" were the main factors that hinder HCPs from reading a DHPC.

\section{RECOMMENDATIONS}

Further research is required on the effectiveness of DHPC on a large sample of HCPs in different governorates of Egypt.

\section{Declarations}

\section{Ethics approval and consent to participate}

The study protocol was revised and approved by the Committee of Ethics, Faculty of Pharmacy, Ain Shams University (serial number: 83)

\section{Consent to publish}

Not applicable

\section{Competing interests}

The authors declare no competing interests exist

\section{Funding Statement}

No funding source was received.

\section{Authors' contributions}

Conceptualization and Writing - review \& editing (MAF, AAS, LME, NAS), Methodology (MAF, AAS, LME), data curation (MAF), Formal analysis (MAF, LME), supervision (LME, AAS, NAS).

\section{REFERENCES}

1. Guideline on good pharmacovigilance practices (GVP) For Arab Countries. 2015. p. 532.(http://www.pharmacovigilance.rns.tn/pdf/GUID ELINE\%20on\%20good\%20pharmacovigilance\%20p ractice\%20for\%20arab\%20countries.pdf). Last accessed: 4-2019.

2. Sarker A, Ginn R, Nikfarjam A, O'Connor K, Smith K, Jayaraman S, Upadhaya T, Gonzalez G. Utilizing social media data for pharmacovigilance: a review. J. Biomed. Inform. 2015;54:202-12.( http://dx.doi.org/10.1016/j.jbi.2015.02.004) 
3. Mammì M, Citraro R, Torcasio G, Cusato G, Palleria $\mathrm{C}$, di Paola ED. Pharmacovigilance in pharmaceutical companies: An overview. JPP. 2013;4(1):S33.(DOI: 10.4103/0976-500X.120945)

4. Sabblah GT, Darko DM, Asamoa-Amoakohene A, Ashie A. The effectiveness of dear healthcare professional letters as a risk minimization tool in Ghana. AJPP. 2016;10(33):681-9.( DOI: 10.5897/AJPP2016.4614)

5. Cupelli A, Baldelli I, Coleman AM, Montero D, Šipić I, Andrić A, Wennberg A, Ahlqvist-Rastad J, Denig P, Mol PG. Communication on Safety of Medicines in Europe: Current Practices and General Practitioners' Awareness and Preferences. Drug Saf. 2017;40(8):729-42.(doi: 10.1007/s40264-017-05350)

6. Desselle, S. P., et al., The effectiveness of written communication for decision support in clinical practice. $\quad$ RSAP, 2019. https://doi.org/10.1016/j.sapharm.2019.06.005

7. Edwards B, Chakraborty S. Risk Communication and the Pharmaceutical Industry. Drug Saf. 2012;35(11):1027-40.(DOI: https://doi.org/10.1007/BF03261989)

8. Schächtele S, Tümena T, Gaßmann KG, Fromm MF, Maas R. Implementation of warnings from Dear Doctor Letters (Rote-Hand-Briefe): an analysis of medication data from a large cohort of elderly patients. DTSCH ARZTEBL INT. 2014;111(15):255.(doi: 10.3238/arztebl.2014.0255)

9. Daniluk J, Cooper JA, Stender M, Kowalczyk A. Survey of physicians' understanding of specific risks associated with retigabine. Drugs Real World Outcomes. 2016;3(2):155-63.(DOI: https://doi.org/10.1007/s40801-016-0068-3)

10. Araujo M., et al., Non-steroidal anti-inflammatory drug prescriptions from the 6th month of pregnancy: impact of advice from health authorities. FUND CLIN PHARMACOL, 2019. https://doi.org/10.1111/fcp.12460

11. Piening S, Haaijer-Ruskamp FM, de Graeff PA, Straus SM, Mol PG. Healthcare professionals' selfreported experiences and preferences related to direct healthcare professional communications. Drug Saf. 2012;35(11):1061-72.(DOI: https://doi.org/10.1007/BF03261992)
12. Lee LY, Kortepeter CM, Willy ME, Nourjah P. Drug-risk communication to pharmacists: assessing the impact of risk-minimization strategies on the practice of pharmacy. J. Am Pharm Association. 2008;48(4):494-500. https://doi.org/10.1331/JAPhA.2008.07045

13. Mazor KM, Andrade SE, Auger J, Fish L, Gurwitz JH. Communicating safety information to physicians: an examination of dear doctor letters. PHARMACOEPIDEM DR S. 2005;14(12):869-75. (DOI: https://doi.org/10.1002/pds.1102)

14. de Vries ST, van der Sar MJ, Coleman AM, Escudero Y, Pascual AR, Martínez MÁ, Cupelli A, Baldelli I, Šipić I, Andrić A, Michan L. Safety communication tools and healthcare professionals' awareness of specific drug safety issues in Europe: a survey study. Drug Saf. 2018;41(7):713-24. (DOI: https://doi.org/10.1007/s40264-018-0643-5)

15. Piening S, de Graeff PA, Straus SM, HaaijerRuskamp FM, Mol PG. The additional value of an email to inform healthcare professionals of a drug safety issue: a randomized controlled trial in the Netherlands. Drug Saf. 2013;36(9):723-31.(DOI: https://doi.org/10.1007/s40264-013-0079-x)

16. Raosoft sample size calculator (http://www.raosoft.com/samplesize.html)

17. Guideline on good pharmacovigilance practices (GVP), Module XVI (Rev 2). 2017, Dover Publications: Mineola, N.Y. p. 22. (https://www.fdanews.com/ext/resources/files/03/ 03-03-14-Pharmacovigilance.pdf). Last accessed: 52019.

18. Guidelines for safety monitoring of medicinal products. $2018 . \quad$ p. 28 . (https://fdaghana.gov.gh/images/stories/pdfs/Safety\% 20Monitoring/2018/4_Guidelines\%20for\%20Safety \%20Monitoring\%20\%20of\%20Products_Feb2018.pd f). Last accessed: 6-2019. 